\title{
All-XUV Pump-Probe Transient Absorption Spectroscopy of the Structural Molecular Dynamics of Di-iodomethane
}

\author{
Marc Rebholz@, ${ }^{1, *}$ Thomas Ding $\odot,{ }^{1}$ Victor Despré $\odot,{ }^{2}$ Lennart Aufleger $\odot,{ }^{1}$ Maximilian Hartmann $\odot,{ }^{1}$ Kristina Meyer, ${ }^{1}$ \\ Veit Stooß@, ${ }^{1}$ Alexander Magunia, ${ }^{1}$ David Wachs, ${ }_{1}^{1}$ Paul Birk $\odot,{ }^{1}$ Yonghao Mi $\odot,{ }^{1}$ Gergana Dimitrova Borisova $\odot,{ }^{1}$ \\ Carina da Costa Castanheira, ${ }^{1}$ Patrick Rupprecht, ${ }^{1}$ Georg Schmid, ${ }^{1}$ Kirsten Schnorr $\odot{ }^{3}$ Claus Dieter Schröter, ${ }^{1}$ \\ Robert Moshammer, ${ }^{1}$ Zhi-Heng Loh $\odot,{ }^{4}$ Andrew R. Attar, ${ }^{5}$ Stephen R. Leone $\odot,{ }^{6,7}$ Thomas Gaumnitz $\odot,{ }^{8}$ Hans \\ Jakob Wörner $\odot,{ }^{8}$ Sebastian Roling, ${ }^{9}$ Marco Butz, ${ }^{9}$ Helmut Zacharias $\odot,{ }^{9}$ Stefan Düsterer $\odot,{ }^{10}$ Rolf Treusch $\odot,{ }^{10}$

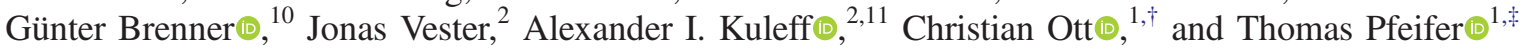 \\ ${ }^{1}$ Max-Planck-Institut für Kernphysik, Saupfercheckweg 1, 69117 Heidelberg, Germany \\ ${ }^{2}$ Theoretische Chemie, Physikalisch-Chemisches Institut, Universität Heidelberg, \\ Im Neuenheimer Feld 229, 69120 Heidelberg, Germany \\ ${ }^{3}$ Paul-Scherrer-Institut, Forschungsstrasse 111, 5232 Villigen, Switzerland \\ ${ }^{4}$ Division of Chemistry and Biological Chemistry, School of Physical and Mathematical Sciences, \\ Nanyang Technological University, Singapore 637371, Singapore \\ ${ }^{5}$ SLAC National Accelerator Laboratory, 2575 Sand Hill Road, Menlo Park, California 94025, USA \\ ${ }^{6}$ Department of Chemistry, University of California, Berkeley, California 94720, USA \\ ${ }^{7}$ Chemical Sciences Division, Lawrence Berkeley National Laboratory, Berkeley, California 94720, USA \\ ${ }^{8}$ Laboratorium für Physikalische Chemie, Eidgenössische Technische Hochschule Zürich, \\ Vladimir-Prelog-Weg 2, 8093 Zürich, Switzerland \\ ${ }^{9}$ Physikalisches Institut, Westfälische Wilhelms-Universität, \\ Wilhelm-Klemm-Straße 10, 48149 Münster, Germany \\ ${ }^{10}$ Deutsches Elektronen-Synchrotron DESY, Notkestraße 85, 22607 Hamburg, Germany \\ ${ }^{11}$ ELI-ALPS, W. Sandner utca 3, 6728 Szeged, Hungary
}

(Received 9 June 2020; revised 27 February 2021; accepted 10 May 2021; published 1 July 2021)

In this work, we use an extreme-ultraviolet (XUV) free-electron laser (FEL) to resonantly excite the I: $4 d_{5 / 2}-\sigma^{*}$ transition of a gas-phase di-iodomethane $\left(\mathrm{CH}_{2} \mathrm{I}_{2}\right)$ target. This site-specific excitation generates a $4 d$ core hole located at an iodine site, which leaves the molecule in a well-defined excited state. We subsequently measure the time-dependent absorption change of the molecule with the FEL probe spectrum centered on the same I: $4 d$ resonance. Using $a b$ initio calculations of absorption spectra of a transient isomerization pathway observed in earlier studies, our time-resolved measurements allow us to assign the timescales of the previously reported direct and indirect dissociation pathways. The presented method is thus sensitive to excited-state molecular geometries in a time-resolved manner, following a core-resonant site-specific trigger.

DOI: 10.1103/PhysRevX.11.031001

\section{INTRODUCTION}

Spectroscopy of core-electron excitations in atoms and molecules has improved our understanding of their structure [1-4]. From a dynamical point of view, the excitation of a core electron in a molecule can trigger many different

\footnotetext{
"marc.rebholz@mpi-hd.mpg.de †christian.ott@mpi-hd.mpg.de

*thomas.pfeifer@mpi-hd.mpg.de
}

Published by the American Physical Society under the terms of the Creative Commons Attribution 4.0 International license. Further distribution of this work must maintain attribution to the author(s) and the published article's title, journal citation, and DOI. Open access publication funded by the Max Planck Society.
Subject Areas: Atomic and Molecular Physics

relaxation mechanisms [5-7]. In addition to purely electronic decay, as occurs in isolated atoms, the relaxation of an excited molecule typically also involves significant changes in its internuclear structure, which may eventually lead to the dissociation of the molecule [8,9].

In recent years, many experiments on femtosecond molecular dynamics were made possible by the advances in ultrashort laser-pulse technology: for example, the spectroscopic investigation of femtosecond photodissociation dynamics initiated by bond breaking with strong-field infrared [10-12] and ultraviolet [8] laser pulses, which has more recently been augmented with site-specific spectroscopy through extreme-ultraviolet (XUV) and soft x-ray light from high-order harmonic generation [13-17].

With the advent of XUV [18] and x-ray free-electron lasers (FELs) [19], new possibilities emerged to investigate 
molecular photodissociation dynamics [20-26]. More specifically, FEL-pump-FEL-probe experiments allows one to resonantly excite and subsequently probe core-to-valence transitions through the interaction with multiple XUV photons, revealing, for instance, the charge-rearrangement dynamics in dissociating molecules [20,25]. Using XUV or $\mathrm{X}$-ray photons, nonperturbative effects such as Stark shifts are strongly suppressed, giving more straightforward access to the field-free molecular structure and dynamics. In combination with the coincidence detection of charged particles, the structural change of molecules could, in this way, be mapped with femtosecond time resolution [27]. Furthermore, the nonlinear interaction with two high-energy photons through a stimulated resonant X-ray Raman process [28-30] can be used to trigger a chemically relevant valence excitation of the molecule, with added site specificity through the localized interaction with a core-shell electron.

For the di-iodomethane $\left(\mathrm{CH}_{2} \mathrm{I}_{2}\right)$ target of interest here, photoinduced structural dynamics have been observed following ultraviolet valence excitation [31,32]. Furthermore, the dissociation and wave-packet dynamics of di-iodomethane initiated by strong-field ionization has been time resolved and measured with site-specific XUV transient absorption spectroscopy [33]. Such structural dynamics typically evolve on the femtosecond timescale.

In contrast to initiating the molecular dynamics through the excitation of a delocalized valence electron with an optical laser, the resonant XUV excitation of a core electron leaves the molecule in a locally excited initial state from which it evolves further. In this work, we combine transient-absorption spectroscopy with a sequence of two femtosecond XUV-FEL pulses to time resolve the ultrafast structural dynamics of di-iodomethane after resonant sitespecific I: $4 d_{5 / 2}-\sigma^{*}$ excitation. Transient absorption spectroscopy hereby allows one to not only detect the charged final states of the molecular fragments, which may decay further on their way to an ion detector, but also to resolve the real-time change of the molecular absorption spectra on the femtosecond timescale.

\section{EXPERIMENT}

The experiment was performed at the self-amplified spontaneous emission (SASE) free-electron laser in Hamburg (FLASH), operated in single-bunch mode at a $10-\mathrm{Hz}$ repetition rate. A sketch of the experimental setup can be found in Fig. 1. With these XUV FEL pulses, we carry out pump-probe transient absorption spectroscopy in a Fraunhofer-type transmission mode. Hereby, the pumpinduced dynamics are detected by scanning the time delay $\Delta t$ between pump and probe pulses and detecting the probe spectrum after transmission through a moderately dense gas target, attenuating the probe transmission according to Beer-Lambert's law. More details on the experimental setup can also be found in Ref. [34]. The incoming FEL beam is spatially cut in two halves using the split-and-delay

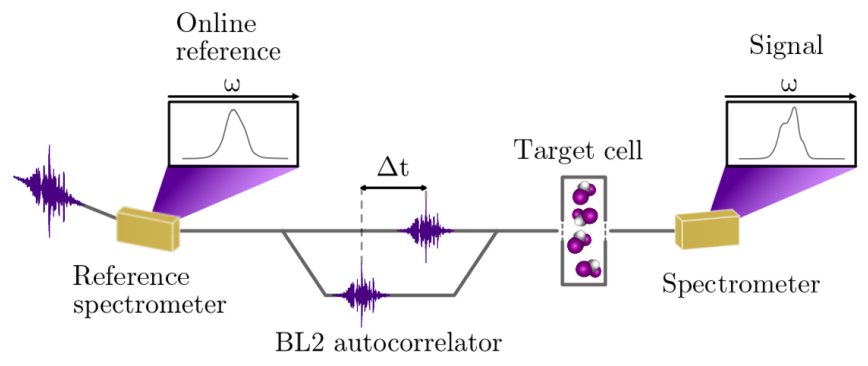

FIG. 1. Sketch of the experimental setup. The incoming FEL spectrum is parasitically measured via the VLS online spectrometer [36]. The main beam then passes the FLASH BL2 autocorrelator [35] to split the beam into pump and probe pulses with a variable time delay $\Delta t$ between them. These pulses are focused into the interaction volume (target cell, filled with gas-phase di-iodomethane), and the probe transmission spectra are detected as a function of the pump-probe time delay.

unit at FLASH Beamline BL2 [35] to generate far-fieldseparated pump and probe pulses. These pulses can be delayed in time with respect to each other by changing the length of one of the two beam paths. The pulses are then focused with an ellipsoidal mirror (2-m focal length), installed at FLASH Beamline BL2, into a cell of 4-mm length filled with gas-phase di-iodomethane at a backing pressure of about $17 \mathrm{mbar}$, which is obtained through gentle heating of the liquid-sample reservoir to about $80^{\circ} \mathrm{C}$. In our experiment, the time delay between the pump and probe pulses is varied between $-400 \mathrm{fs}$ and $+800 \mathrm{fs}$. After interaction with the target, the probe pulse is spectrally resolved in a flat-field spectrometer with a resolution below $35 \mathrm{meV}$ at $50 \mathrm{eV}$. The spectrometer consists of a variable-linespacing (VLS) grazing-incidence concave diffraction grating, together with an XUV-CCD camera. Moreover, because of the far-field separation of pump and probe pulses, the dynamical information encoded in the probe spectra is detected separately from the pump. To determine the absorbance of the FEL pulses after interaction with the diiodomethane target, the FLASH VLS online spectrometer [36] is used to record reference spectra while performing the experiment. In this configuration, one mirror of the FEL beamline is replaced by a flat VLS diffraction grating. Its first diffraction order is used as a reference spectrum, while it acts as a plane mirror for the zeroth order, which is propagated further downstream to the experiment. For every time-delay setting, the probe spectra of 100 individual pulses are recorded, together with the corresponding online reference spectra. The absorbance is then calculated according to Beer-Lambert's law by using the average over each of the 100 pulses.

For the temporal characterization of the FEL pulses and to determine the time resolution of the experiment, a secondorder autocorrelation in neon is performed with the same machine settings [37]. The temporal instrument response function (IRF) is measured at $(65 \pm 15)$ fs FWHM (for details, see Supplemental Material [38]). Furthermore, the 
temporal overlap of pump and probe pulses is obtained with an accuracy below $10 \mathrm{fs}$ through the coherent enhancement of transmission when individual temporal modes of the noisy SASE FEL pulse precisely overlap in time $[37,40]$. The average FEL pulse energy of $44 \mu \mathrm{J}$ (standard deviation 29\%, over an ensemble of 2500 pulses) - measured upstream with the FLASH Beamlines gas monitor detector [41], an ontarget focal spot size of about $25 \mu \mathrm{m}$, and a FEL average pulse duration of $65 \mathrm{fs}$, combined with the beamline transmission of the given setup of roughly $25 \%$ - translates to an average on-target intensity in the $10^{13} \mathrm{~W} \mathrm{~cm}^{-2}$ regime being an order-of-magnitude estimate.

By tuning the FEL photon energy to the I: $4 d_{5 / 2}-\sigma^{*}$ transition of di-iodomethane at $50.4 \mathrm{eV}$, we resonantly excite a localized iodine $4 d$ electron into an antibonding molecular orbital in the valence shell. The generation of this core hole leads to resonant Auger decay on a timescale of about $10 \mathrm{fs}$ $[42,43]$, which is much faster than the given instrument response; during this time, the nuclei have not yet moved significantly along their dissociating pathways. Thus, the cationic states populated after this Auger decay further determine the subsequent structural molecular dynamics. Since the majority of the Auger final states are energetically well below the threshold for double ionization, the singly charged cation $\left(\mathrm{CH}_{2} \mathrm{I}_{2}{ }^{+}\right)$is produced as the most dominant

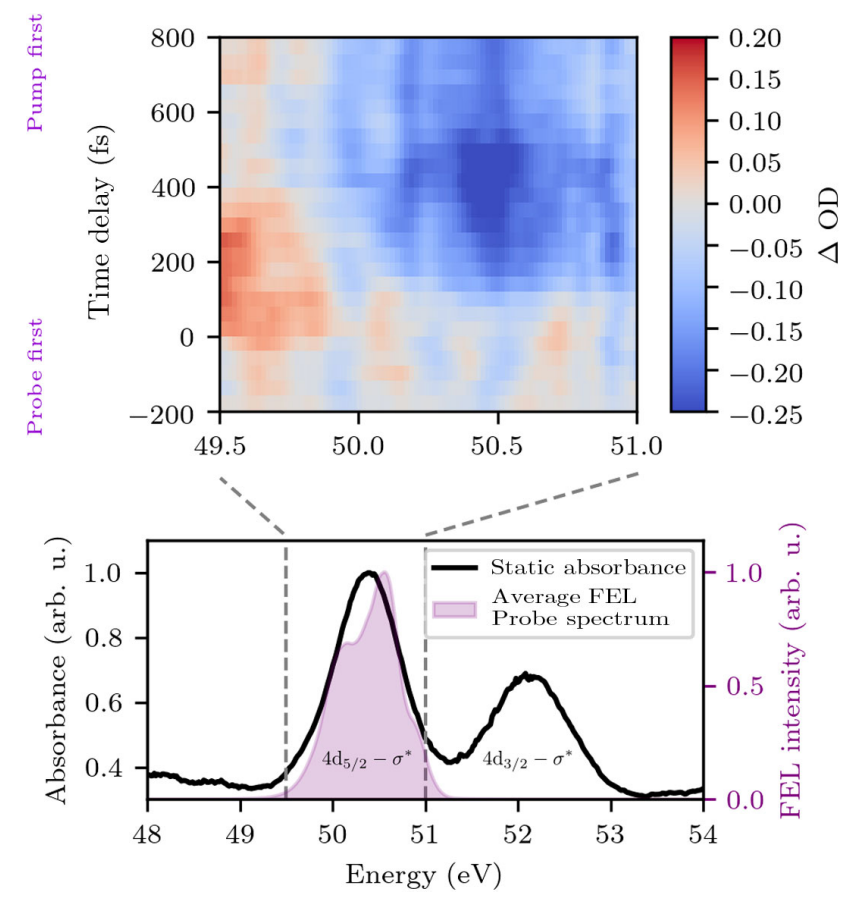

FIG. 2. Measured $\Delta \mathrm{OD}$ around the I: $4 d_{5 / 2}-\sigma^{*}$ transition of di-iodomethane over the course of the pump-probe time delay (upper panel). In the lower panel, the static absorbance of di-iodomethane is shown, measured with a broadband XUV high-harmonic source over a wider energy range, where the spinorbit splitting of the transition is visible. The average FEL probe spectrum with respect to the static absorbance is illustrated by violet shading. species, represented by an ensemble of only a few excited states [44], while the Auger electron carries away the remaining excess energy (see Supplemental Material [38] for more details). The subsequent molecular relaxation dynamics, i.e., the dissociating pathways, are probed via the I: $4 d$ core-level absorption spectra of the cation as a function of the pump-probe time delay.

The change in absorbance ( $\Delta \mathrm{OD})$ of the probe pulse after transmission through di-iodomethane is shown in Fig. 2 as a function of the time delay with respect to the pump pulse. It is obtained by subtracting the absorbance for very early delays, averaged from -400 fs to $-100 \mathrm{fs}$. It can be clearly seen that the absorbance on resonance decreases smoothly for positive delays.

\section{GLOBAL ANALYSIS}

To quantify the associated timescales of the change in absorption, different photon-energy regions are spectrally integrated. For the individual energy regions, we observe a qualitatively and quantitatively different behavior (see Fig. 3 and Table I). In the low-energy region between $49.5 \mathrm{eV}$ and $49.8 \mathrm{eV}$, a rapid increase and subsequent decay are observed. On resonance (between $50.0 \mathrm{eV}$ and $50.8 \mathrm{eV}$ ), the absorption decreases smoothly. The error bars are calculated by taking the standard error of the mean for the $100 \mathrm{FEL}$ spectra recorded for each time-delay setting. In order to quantify the timescale of the dynamics, a global exponential fit is employed:

$$
\begin{aligned}
\Delta \mathrm{OD}(E, t)= & y(E)+\operatorname{IRF}(t) *\left[\Theta(t) \times\left(\Delta \mathrm{OD}_{\text {late }}(E)\right.\right. \\
& \left.\left.+\Delta A_{1}(E) e^{-t / \tau_{1}}+\Delta A_{2}(E) e^{-t / \tau_{2}}\right)\right] .
\end{aligned}
$$

Here, $y(E)$ is a time-independent offset, $\operatorname{IRF}(t)$ is the instrument response function, $\Theta(t)$ is the Heaviside step

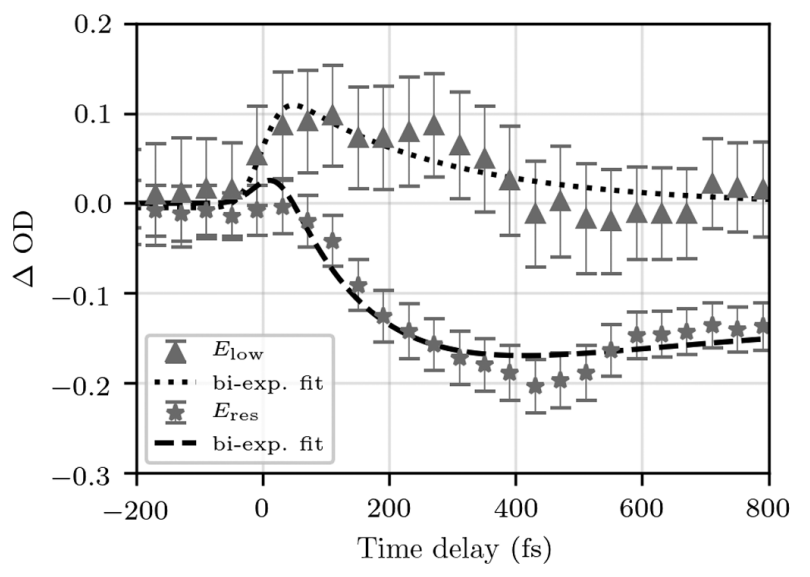

FIG. 3. $\Delta \mathrm{OD}$ integrated over different spectral regions. In the low-energy range ( $E_{\text {low }}$ from 49.5 to $49.8 \mathrm{eV}$ ), an initial rise with a subsequent decay can be seen. Integrating over the resonance $\left(E_{\text {res }}\right.$ from 50.0 to $50.8 \mathrm{eV}$ ), the $\Delta \mathrm{OD}$ shows a smooth decrease. Global fitting of an exponential model [Eq. (1)] to these lineouts reveals the timescales $\tau_{1}=(166 \pm 11)$ fs and $\tau_{2}=(290 \pm 30)$ fs. 
TABLE I. Results of the global exponential fit [Eq. (1)] to the data.

\begin{tabular}{llc}
\hline Energy region $(\mathrm{eV})$ & $\tau_{1}=(166 \pm 11)$ fs & $\tau_{2}=(290 \pm 30)$ fs \\
\hline$E_{\text {low }}: 49.5-49.8$ & $\Delta A_{1}=0.04 \pm 0.06$ & $\Delta A_{2}=0.11 \pm 0.05$ \\
$E_{\text {res }}: 50.0-50.8$ & $\Delta A_{1}=0.57 \pm 0.06$ & $\Delta A_{2}=-0.34 \pm 0.05$ \\
\hline \hline
\end{tabular}

function, and $\Delta \mathrm{OD}_{\text {late }}(E)$ is the differential absorbance for large positive delays $(t=800 \mathrm{fs})$. The $*$ denotes the convolution between the instrument response function and the function describing the dynamics. The time constants $\tau_{1}$ and $\tau_{2}$ quantify the underlying dynamics of the absorption change with the corresponding spectral weights $\Delta A_{1}(E)$ and $\Delta A_{2}(E)$. Because of the spectrally narrow probe window of $1.5 \mathrm{eV}$ and general limitations due to SASE FEL fluctuations, we restrict the global fit to two spectral components on resonance $\left(E_{\text {res }}\right)$ and at low photon energies $\left(E_{\text {low }}\right)$ instead of the entire spectrotemporal absorption trace. The outcome of the global fit is summarized in Table I, extracting the time constants $\tau_{1}=(166 \pm 11)$ fs and $\tau_{2}=(290 \pm 30)$ fs, and the resulting curves are plotted in Fig. 3.

\section{STRUCTURAL ANALYSIS}

The transient increase in absorption in the low-energy region $E_{\text {low }}$ indicates that, in addition to the direct dissociation of the $\mathrm{CH}_{2} \mathrm{I}_{2}{ }^{+}$molecular ion, another relaxation mechanism may be at work. Indeed, a transient isomeric species, $\mathrm{CH}_{2} \mathrm{I}-\mathrm{I}^{+}$, has been identified through static synchrotron measurements [45] to play a role in the XUV-initiated dissociation pathways of $\mathrm{CH}_{2} \mathrm{I}_{2}{ }^{+}$. We pursue this possibility by generating and using theoretically predicted spectra of different isomeric molecular structures in order to investigate if the observed features in our measurement are compatible with this relaxation mechanism, and thus assign the corresponding timescales. We calculate the XUV excitation spectra of $\mathrm{CH}_{2} \mathrm{I}_{2}{ }^{+}$after Auger decay by using the $a b$ initio algebraic diagrammatic construction Green's function method at third order [ADC(3)] [46]. The good accuracy of this method has been demonstrated in a large number of benchmark calculations [47,48]. It is especially suitable for the treatment of electron correlation effects and multielectron excitation, which makes it the method of choice for describing deep inner-valence and outer-valence excited ionic states of molecules. Unfortunately, also including the structural dynamics of the molecule from an ab initio perspective is currently out of reach. Therefore, our model incorporates existing knowledge of the possible structural pathways in order to (i) check whether these pathways can also be used to explain the observations in our measurement and (ii) explicitly time resolve the underlying dynamics.

The ADC(3) calculations yielding the cationic excitation spectra are performed for different molecular geometries that represent selected minimum-energy structures along the nontrivial dissociation path of the $\mathrm{CH}_{2} \mathrm{I}_{2}{ }^{+}$cationic ground state $[45,49]$. We compute the transition dipole moments between the first ten cationic excited states, which are populated after the resonant Auger decay, and all higher lying states that can be reached by the absorption of a single XUV probe photon at about $50 \mathrm{eV}$. The absorption spectra of the cation are then calculated for the different geometries by weighting every transition with the absolute value squared of its corresponding transition dipole moment and convoluting it with a Gaussian, the width of which corresponds to the Auger lifetime (for details, see Supplemental Material [38]). The resulting theoretical absorption spectra for the different cationic molecular geometries are depicted in Fig. 4, together with the measured absorption spectrum of the neutral molecule.

Towards a lower photon energy of around $49.5 \mathrm{eV}$, geometry 2 is the dominating species of these proposed molecular structures. Its absorption peak coincides with the transient feature that is observed in the experimental data (cf. Fig. 2), which is a first hint that this experimentally observed transient feature can be identified with a corresponding $\mathrm{CH}_{2} \mathrm{I}-\mathrm{I}^{+}$isomeric geometry, similar to geometry 2 (see Supplemental Material [38]). In order to further substantiate this assignment, we perform an additional measurement on the XUV-induced dissociation dynamics
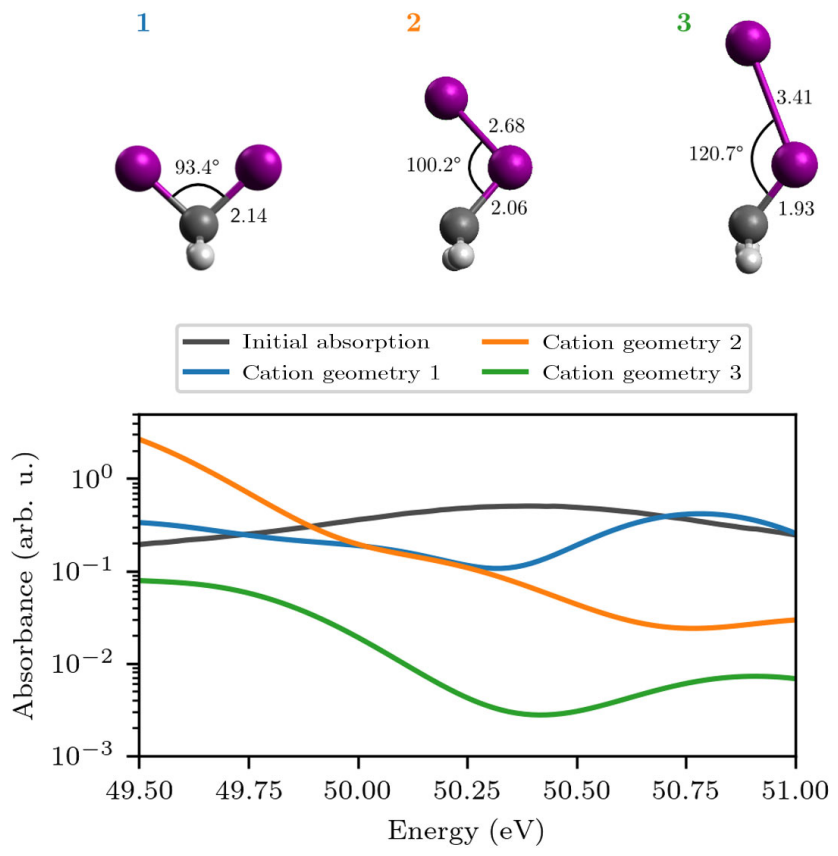

FIG. 4. Theoretically calculated absorption spectra for the different geometries of the di-iodomethane cation, as well as the measured absorption spectrum of neutral di-iodomethane. Note the logarithmic scale. Geometry 1 represents the arrangement of the nuclei in the cationic ground state. Geometries 2 and 3 are the minimum-energy structures along the molecule's dissociation path passing through the isomeric geometry (numerical input data for the different geometries were obtained from Ref. [45]). All distances are given in $\AA$. 
of methyl iodide $\mathrm{CH}_{3} \mathrm{I}$ under similar experimental conditions (see Supplemental Material [38] for details). In the experiment, we do not observe a significant transient increase in absorbance for this target species. Furthermore, we have also calculated the theoretical absorption spectra for the dissociating $\mathrm{CH}_{2} \mathrm{I}_{2}{ }^{+}$molecular ion for additional molecular geometries over a broader probing bandwidth (see Supplemental Material [38]). From these spectra, we can identify a dominant peak at low photon energy that appears for cases when a bond between the two iodine sites can be formed. Thus, both from an experimental and from a theoretical point of view, we find evidence for the relevance of a transient isomeric geometry in the dissociation of the $\mathrm{CH}_{2} \mathrm{I}_{2}{ }^{+}$molecular ion in our measurement, in agreement with previous findings for a similar excitation scenario [45].

With these spectra in hand, we now turn to a more quantitative analysis of the time-resolved changes. During the dissociation process, the molecular cation may transiently pass through different isomeric geometries, which are approximated here by geometries $1-3$, before breaking apart. Therefore, the total absorption spectrum is expected to contain time-dependent contributions from these molecular geometries of varying strength. The differential absorbance $\Delta \mathrm{OD}$ can then be straightforwardly modeled as

$$
\begin{aligned}
\Delta \mathrm{OD}(E, t)= & \Delta a_{0}(t) S_{0}(E)+\Delta a_{1}(t) S_{1}(E) \\
& +\Delta a_{2}(t) S_{2}(E)+\Delta a_{3}(t) S_{3}(E)+b(t) .
\end{aligned}
$$

Here, the $S_{i}(E)$ denote the absorption spectra of the individual cationic geometries, while the amplitudes $\Delta a_{i}(t)$ are their contributions to the differential absorption over the course of the time delay and, therefore, reflect the change in the molecular geometry, for $i=1,2,3$. Note that $S_{0}(E)$ denotes the absorption spectrum of the initial cationic state in the geometry of the neutral molecule, which we assume to closely resemble the measured absorption spectrum of the latter, with its contribution $\Delta a_{0}(t)$ to the differential absorption. The residual nonresonant background absorption is accounted for by a time-dependent offset $b(t)$. The function given in Eq. (2) is fitted to the experimental data for every time-delay step by varying the coefficients $\Delta a_{j}(t)$, with $j=0,1,2,3$, and minimizing the squared residuals. The resulting time-dependent evolution of the molecular states can be found in Fig. 5.

\section{DISCUSSION}

The progression of the molecular cation through its different isomeric geometries can be clearly seen to follow a logical time ordering of $\Delta a_{0}(t), \Delta a_{1}(t), \Delta a_{2}(t)$, and $\Delta a_{3}(t)$. This progression further confirms the validity of assigning the suggested nontrivial dissociation pathway through, or close by, the minimum-energy structures 1-3 to explain the measured data. The cationic ground-state

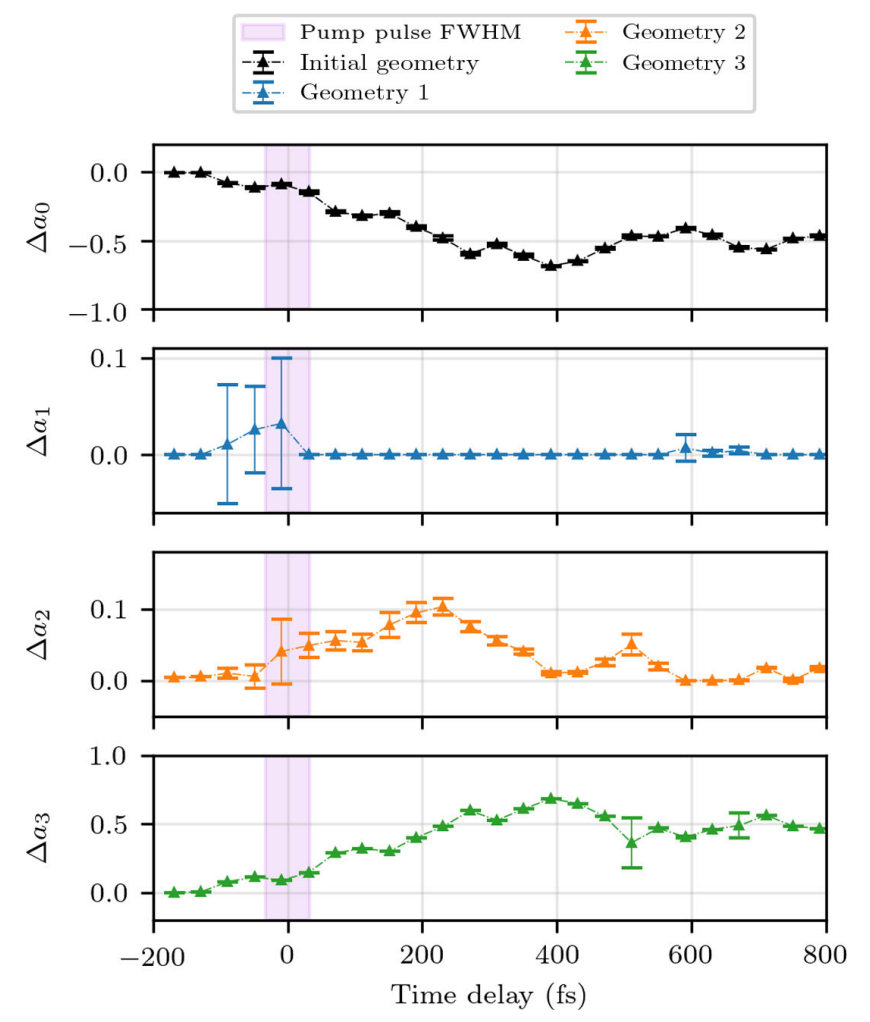

FIG. 5. Contributions of the different configurations of the target to the total absorption. The purple shaded area illustrates the duration (FWHM) of the pump pulse. The amplitudes $\Delta a_{i}$ are the results of fitting Eq. (2) to the experimental data. The error bars depict the fit error. Here, $\Delta a_{0}$ shows the temporal evolution of the cationic initial geometry, with the nuclei being at their respective positions in the neutral molecule, and $\Delta a_{1}, \Delta a_{2}$, and $\Delta a_{3}$ represent the absorption contributions from the cationic geometries as drawn in Fig. 4.

geometry 1 does not effectively contribute to the measured changes in absorption for all times [cf. $\Delta a_{1}(t)$ ], while we identify only a minor contribution at zero time delay, where the proximity to the geometric ground state of the cation is assumed to be highest. Instead, the cation is initially prepared in the ground-state geometry of the neutral molecule since the nuclei did not have time to move significantly within the XUV excitation and successive Auger decay. Hereby, the Auger lifetime is far below the temporal resolution of the measurement and thus safe to be considered as instantaneous. At later times, the bleach of this initial state is thus observed by a gradual decrease of $\Delta a_{0}(t)$. When the structure of the cation resembles the isomeric geometry 2, a transient increase and decrease is observed in $\Delta a_{2}(t)$. Interestingly, a local maximum of this contribution is observed just after $200 \mathrm{fs}$, which, upon closer inspection, can also be identified in the low-energy region of Fig. 3. Finally, a gradual increase of geometry 3 is observed, which saturates at about $400 \mathrm{fs}$ after the temporal overlap. Based on the complete decrease of the initial-state geometry and the transient geometry 2 at very late times, one may conclude that the dissociation process has only 
been completed after about 400 fs. Further deviations, especially at late time delays, including the increase of absorption in the resonant energy region, are likely caused by the exclusion of the molecular fragments in our theoretical treatment. Their contribution is expected to become increasingly important at late times; however, including even more channels is not possible for the current data set given the limited spectral probing window.

For better comparability to the experimentally measured data (Fig. 2), the fit amplitudes $\Delta a_{j}$, with $j=0,1,2,3$ (see Fig. 5), together with the corresponding absorption spectra (Fig. 4), are used to model $\Delta \mathrm{OD}^{(\mathrm{rec})}(E, t)$ (Fig. 6). Overall, the main features of the experiment, namely, the resonant decrease of absorption in the vicinity of $E_{\text {res }}$, as well as the transient increase and subsequent decay of the absorption signal in the low-energy region $\left(E_{\text {low }}\right)$, are reproduced very well. Additionally, in Fig. 7, lineouts of the reconstructed $\Delta \mathrm{OD}_{0,2}^{(\mathrm{rec})}(E, t)$ are shown, which, in this case, are generated by only including specific contributions of the initial geometry $\left[\Delta a_{0}(t) \times S_{0}(\omega)+b(t)\right]$, respectively geometry $2\left[\Delta a_{2}(t) \times S_{2}(\omega)\right]$, setting all others to zero. The lineouts are taken through the lower energy region $\left(E_{\text {low }}\right)$ for geometry 2 and the resonant energy region $\left(E_{\text {res }}\right)$ for the initial geometry. It is evident that the decrease on resonance is dominated by the bleaching of the initial geometry, while the transient feature in the low-energy region $\left(E_{\text {low }}\right)$ is dominated by a molecular structure similar to geometry 2 (see Supplemental Material [38]). To make the connection to the bi-exponential fit [Eq. (1)] of the measured $\Delta \mathrm{OD}(E, t)$, the content of Fig. 3 is reproduced in the background of Fig. 7. The observed dynamics can be explained by two processes occurring in parallel. One process is the direct dissociation of the di-iodomethane cation, which mainly manifests as the resonant decrease $\left(E_{\text {res }}\right)$ of absorption and is linked to a decrease of the molecule's initial geometry. The other process is the indirect dissociation involving an isomeric geometry of the cation, which leads to the transient feature in the lower-

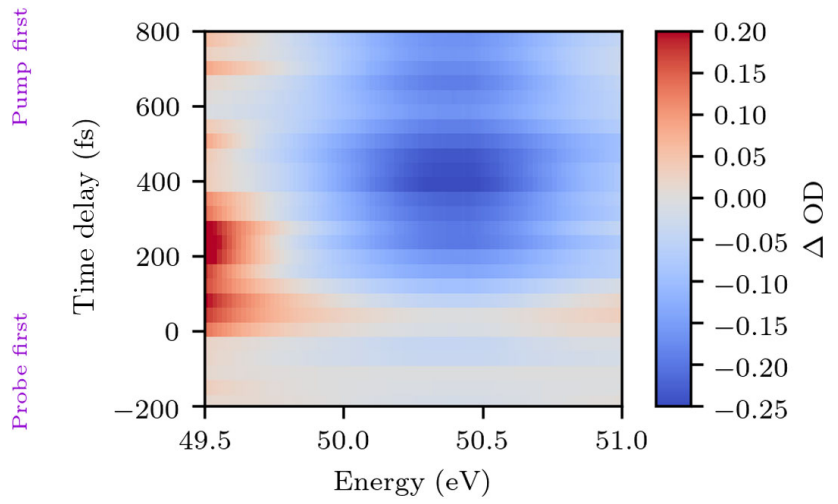

FIG. 6. $\Delta \mathrm{OD}^{(\mathrm{rec})}(E, t)$, reconstructed from the absorption spectra $S_{j}(E)$ and their contributions to the total absorbance $\Delta a_{j}(t)(j=0,1,2,3)$, which are obtained from fitting Eq. (2) to the experimentally measured data.

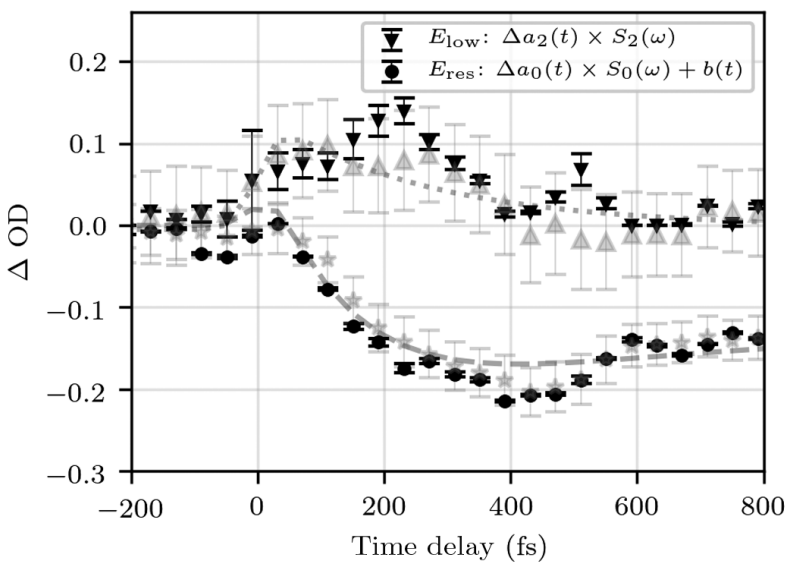

FIG. 7. Lineouts through the resonant energy region $\left(E_{\text {res }}\right)$ and the low-energy region $\left(E_{\text {low }}\right)$ of the reconstructed $\Delta \mathrm{OD}_{0,2}^{(\mathrm{rec})}(E, t)$ with only specific channels $\left(a_{0}\right.$, respectively $\left.a_{2}\right)$ included. All other contributions are set to zero. For comparison, the experimental $\Delta \mathrm{OD}(E, t)$ trace with its bi-exponential fit [cf. Fig. 3 and Eq. (1)] is shown in the background.

energy region $\left(E_{\text {low }}\right)$. As a result of this analysis, we find that this transient feature emerges more slowly than the IRF and approximately decays with the longer time constant of $290 \mathrm{fs}$ revealed by the bi-exponential fit [Eq. (1)]. It has to be noted that the ensemble of multiple excited states (created by the pump pulse) and their pathways all contribute, on average, in this spectral range. The temporal evolution of the molecule's initial geometry and its isomeric geometry 2, identified through the cation's electronic ground state according to Ref. [45], hereby largely suffice to explain the main features of the measured $\Delta \mathrm{OD}(E, t)$ (cf. Fig. 7). Hence, we are able to reveal, in part, the dynamics of a previously known relaxation pathway of this site-specifically excited molecule, while other structural dynamics mediated by the higher-lying electronic states and even the nonadiabatic coupling in between these states may further contribute. The reduced $\Delta \mathrm{OD}_{0,2}^{(\mathrm{rec})}(E, t)$ shows additional features (deviations) as compared to the global fit, which is understandable, as the averaging over multiple decaying pathways in the experiment is removed by considering only a single channel. We note that previous studies on the ultrafast dynamics of diiodomethane, albeit with a different excitation mechanism, have also suggested the coexistence of parallel molecular dissociation pathways [50]. The presented method will clearly benefit from a broader XUV probe spectrum, which is expected to become available in the near future [51-53]. The sensitivity to different molecular structures and charged fragments when using such broadband XUV probing is illustrated in the Supplemental Material [38]. There, we apply the fit model [Eq. (2)] to a simulated absorption trace with a broader spectral range. Probing over a broader photon-energy bandwidth would thus help to differentiate between different molecular geometries and fragments that exhibit distinct spectroscopic features. 
In this work, we have demonstrated that employing ultrashort all-XUV FEL pulses in a transient-absorption spectroscopy experiment is a powerful tool to access XUVinitiated transient femtosecond molecular dynamics. Given the high flux of the FEL radiation, it is possible to perform site-specific XUV-pump-XUV-probe transient-absorption spectroscopy, here applied near the iodine $4 d$ resonance. Supported by $a b$ initio calculations of the XUV electronic excitation spectra for different molecular geometries, we time resolve the structural changes along previously known dissociation pathways. The results have been obtained despite using a relatively narrow probe spectrum of the FEL, which, in our case, is on the order of $0.5 \mathrm{eV} \mathrm{FWHM,}$ typically $1 \%$ of the given photon energy [54]. In the future, we expect this limitation to be overcome by combining a high-order harmonic XUV source as a broadband probe, spanning multiple tens of $\mathrm{eV}$, while retaining the sitespecific XUV excitation with the FEL pump pulse [51]. Furthermore, access to a broad bandwidth of XUV excitations is also enabled by novel two-color FEL operation modes [52,53], and attosecond XFEL pulses are becoming available [55]. Such schemes would enable the detection and spectroscopic identification of a much wider range of final states of the molecular target, including charged and neutral molecular and atomic fragments [12,33], unleashing the full potential of all-XUV transient absorption spectroscopy. While our current work represents a key step, with local pump and local probe, but limited to the same atomic species, the full power of the method will unfold when pump and probe pulses of different photon energies can be used for exciting and observing spectral changes on different atomic species in molecules.

For their support during the experiment, we thankfully acknowledge the technical and scientific staff at FLASH. We gratefully acknowledge C. Kaiser and B. Knape for their technical support. We acknowledge funding from the European Research Council (ERC) (X-MuSiC-616783). Z.-H. L. acknowledges support from the Ministry of Education, Singapore (RG105/17 and RG1/20). V. D. and A. I. K. acknowledge the financial support of DFG through QUTIF Priority Programme. S. R. L. acknowledges the support of the GPCP and AMOS programs of the Division of Chemical Sciences, Geosciences, and Biosciences of the U.S. Department of Energy at LBNL under Contract No. DEAC02-05CH11231 and the National Science Foundation under Grant No. CHE-1660417. H. Z. acknowledges support by the BMBF (Project No. 05K13PM2).

[1] K. Siegbahn, Electron Spectroscopy for Atoms, Molecules, and Condensed Matter, Science 217, 111 (1982).

[2] I. Nenner, P. Morin, P. Lablanquie, M. Simon, N. Levasseur, and P. Millie, Photodissociation of Core Excited Molecules, J. Electron Spectrosc. Relat. Phenom. 52, 623 (1990).
[3] G. O’Sullivan, C. McGuinness, J. T. Costello, E. T. Kennedy, and B. Weinmann, Trends in 4d-Subshell Photoabsorption Along the Iodine Isonuclear Sequence: $\mathrm{I}^{+} \mathrm{I}^{+}$, and $\mathrm{I}^{2+}$, Phys. Rev. A 53, 3211 (1996).

[4] F. J. Comes, U. Nielsen, and W. H. E. Schwarz, Inner Electron Excitation of Iodine in the Gaseous and Solid Phase, J. Chem. Phys. 58, 2230 (1973).

[5] L.S. Cederbaum, J. Zobeley, and F. Tarantelli, Giant Intermolecular Decay and Fragmentation of Clusters, Phys. Rev. Lett. 79, 4778 (1997).

[6] K. Ueda, Core Excitation and De-excitation Spectroscopies of Free Atoms and Molecules, J. Phys. Soc. Jpn. 75, 032001 (2006).

[7] M. N. Piancastelli, R. Guillemin, T. Marchenko, L. Journel, O. Travnikova, T. Marin, G. Goldsztejn, B. C. D. Miranda, I. Ismail, and M. Simon, New Achievements on Relaxation Dynamics of Atoms and Molecules Photoexcited in the Tender X-Ray Domain at Synchrotron SOLEIL, J. Phys. B 50, 042001 (2017).

[8] M. J. Rosker, M. Dantus, and A. H. Zewail, Femtosecond Real-Time Probing of Reactions. I. The Technique, J. Chem. Phys. 89, 6113 (1988).

[9] A. H. Zewail, Femtochemistry: Atomic-Scale Dynamics of the Chemical Bond, J. Phys. Chem. A 104, 5660 (2000).

[10] Z. Wei, J. Li, L. Wang, S. T. See, M. H. Jhon, Y. Zhang, F. Shi, M. Yang, and Z.-H. Loh, Elucidating the Origins of Multimode Vibrational Coherences of Polyatomic Molecules Induced by Intense Laser Fields, Nat. Commun. 8, 735 (2017).

[11] Y. Pertot, C. Schmidt, M. Matthews, A. Chauvet, M. Huppert, V. Svoboda, A. Von Conta, A. Tehlar, D. Baykusheva, J. P. Wolf, and H. J. Wörner, Time-Resolved X-Ray Absorption Spectroscopy with a Water-Window High-Harmonic Source, Science 355, 264 (2017).

[12] Y. Kobayashi, K. F. Chang, T. Zeng, D. M. Neumark, and S. R. Leone, Direct Mapping of Curve-Crossing Dynamics in IBr by Attosecond Transient Absorption Spectroscopy, Science 365, 79 (2019).

[13] A. R. Attar, A. Bhattacherjee, and S. R. Leone, Direct Observation of the Transition-State Region in the Photodissociation of $\mathrm{CH}_{3} \mathrm{I}$ by Femtosecond Extreme Ultraviolet Transient Absorption Spectroscopy, J. Phys. Chem. Lett. 6, 5072 (2015).

[14] L. Drescher, M. C. Galbraith, G. Reitsma, J. Dura, N. Zhavoronkov, S. Patchkovskii, M. J. Vrakking, and J. Mikosch, Communication: XUV Transient Absorption Spectroscopy of Iodomethane and Iodobenzene Photodissociation, J. Chem. Phys. 145, 011101 (2016).

[15] M. Galbraith, S. Scheit, N. Golubev, G. Reitsma, N. Zhavoronkov, V. Despré, F. Lépine, A. Kuleff, M. Vrakking, O. Kornilov, H. Köppel, and J. Mikosch, Few-Femtosecond Passage of Conical Intersections in the Benzene Cation, Nat. Commun. 8, 1018 (2017).

[16] A. Baumann, D. Rompotis, O. Schepp, M. Wieland, and M. Drescher, Time-Resolved Dissociation Dynamics of Iodomethane Resulting from Rydberg and Valence Excitation, J. Phys. Chem. A 122, 4779 (2018).

[17] L. Drescher, G. Reitsma, T. Witting, S. Patchkovskii, J. Mikosch, and M. J. Vrakking, State-Resolved Probing of 
Attosecond Timescale Molecular Dipoles, J. Phys. Chem. Lett. 10, 265 (2019).

[18] W. Ackermann et al., Operation of a Free-Electron Laser from the Extreme Ultraviolet to the Water Window, Nat. Photonics 1, 336 (2007).

[19] B. W. J. McNeil and N. R. Thompson, X-Ray Free-Electron Lasers, Nat. Photonics 4, 814 (2010).

[20] K. Schnorr, A. Senftleben, M. Kurka, A. Rudenko, G. Schmid, T. Pfeifer, K. Meyer, M. Kübel, M. F. Kling, Y. H. Jiang, R. Treusch, S. Düsterer, B. Siemer, M. Wöstmann, H. Zacharias, R. Mitzner, T. J. M. Zouros, J. Ullrich, C. D. Schröter, and R. Moshammer, Electron Rearrangement Dynamics in Dissociating $\mathrm{I}_{2}^{n+}$ Molecules Accessed by Extreme Ultraviolet Pump-Probe Experiments, Phys. Rev. Lett. 113, 073001 (2014).

[21] B. Erk, R. Boll, S. Trippel, D. Anielski, L. Foucar, B. Rudek, S. W. Epp, R. Coffee, S. Carron, S. Schorb, K. R. Ferguson, M. Swiggers, J. D. Bozek, M. Simon, T. Marchenko, J. Küpper, and I. Schlichting, Imaging Charge Transfer in Iodomethane upon X-Ray Photoabsorption, Science 345, 288 (2014).

[22] K. Motomura et al., Charge and Nuclear Dynamics Induced by Deep Inner-Shell Multiphoton Ionization of $\mathrm{CH}_{3} \mathrm{I}$ Molecules by Intense X-Ray Free-Electron Laser Pulses, J. Phys. Chem. Lett. 6, 2944 (2015).

[23] A. Rudenko and D. Rolles, Time-Resolved Studies with FELs, J. Electron Spectrosc. Relat. Phenom. 204, 228 (2015).

[24] A. Picón et al., Hetero-Site-Specific X-Ray Pump-Probe Spectroscopy for Femtosecond Intramolecular Dynamics, Nat. Commun. 7, 11652 (2016).

[25] M. Hollstein, K. Mertens, S. Klumpp, N. Gerken, S. Palutke, I. Baev, G. Brenner, S. Dziarzhytski, M. Meyer, W. Wurth, D. Pfannkuche, and M. Martins, Ultrafast Charge Redistribution in Small Iodine Containing Molecules, New J. Phys. 21, 033017 (2019).

[26] H. Fukuzawa et al., Real-Time Observation of X-RayInduced Intramolecular and Interatomic Electronic Decay in $\mathrm{CH}_{2} \mathrm{I}_{2}$, Nat. Commun. 10, 2186 (2019).

[27] Y. H. Jiang, A. Rudenko, O. Herrwerth, L. Foucar, M. Kurka, K. U. Kühnel, M. Lezius, M. F. Kling, J. Van Tilborg, A. Belkacem, K. Ueda, S. Düsterer, R. Treusch, C. D. Schröter, R. Moshammer, and J. Ullrich, Ultrafast Extreme Ultraviolet Induced Isomerization of Acetylene Cations, Phys. Rev. Lett. 105, 263002 (2010).

[28] C. Weninger, M. Purvis, D. Ryan, R. A. London, J. D. Bozek, C. Bostedt, A. Graf, G. Brown, J. J. Rocca, and N. Rohringer, Stimulated Electronic X-Ray Raman Scattering, Phys. Rev. Lett. 111, 233902 (2013).

[29] J. T. O'Neal et al., Electronic Population Transfer via Impulsive Stimulated X-Ray Raman Scattering with Attosecond Soft-X-Ray Pulses, Phys. Rev. Lett. 125, 073203 (2020).

[30] U. Eichmann et al., Photon-Recoil Imaging: Expanding the View of Nonlinear X-Ray Physics, Science 369, 1630 (2020).

[31] V. A. Borin, S. M. Matveev, D. S. Budkina, P. Z. El-Khoury, and A. N. Tarnovsky, Direct Photoisomerization of $\mathrm{CH}_{2} \mathrm{I}_{2}$ vs. $\mathrm{CHBr}_{3}$ in the Gas Phase: A Joint $50 \mathrm{fs}_{\text {s Experimental and }}$
Multireference Resonance-Theoretical Study, Phys. Chem. Chem. Phys. 18, 28883 (2016).

[32] M. R. Panman et al., Observing the Structural Evolution in the Photodissociation of Diiodomethane with Femtosecond Solution X-Ray Scattering, Phys. Rev. Lett. 125, 226001 (2020).

[33] Z. Wei, J. Li, H. Zhang, Y. Lu, M. Yang, and Z.-H. Loh, Ultrafast Dissociative Ionization and Large-Amplitude Vibrational Wave Packet Dynamics of Strong-Field-Ionized Di-iodomethane, J. Chem. Phys. 151, 214308 (2019).

[34] T. Ding, M. Rebholz, L. Aufleger, M. Hartmann, V. Stooß, A. Magunia, P. Birk, G. D. Borisova, C. da Costa Castanheira, P. Rupprecht, Y. Mi, T. Gaumnitz, Z. Loh, S. Roling, M. Butz, H. Zacharias, S. Düsterer, R. Treusch, C. Ott, and T. Pfeifer, XUV Pump-XUV Probe Transient-Absorption Spectroscopy at FELs, Faraday Discuss. 228, 519 (2021).

[35] M. Wöstmann, R. Mitzner, T. Noll, S. Roling, B. Siemer, F. Siewert, S. Eppenhoff, F. Wahlert, and H. Zacharias, The XUV Split-and-Delay Unit at Beamline BL2 at FLASH, J. Phys. B 46, 164005 (2013).

[36] G. Brenner, S. Kapitzki, M. Kuhlmann, E. Ploenjes, T. Noll, F. Siewert, R. Treusch, K. Tiedtke, R. Reininger, M. D. Roper, M. A. Bowler, F. M. Quinn, and J. Feldhaus, First Results from the Online Variable Line Spacing Grating Spectrometer at FLASH, Nucl. Instrum. Methods Phys. Res., Sect. A 635, S99 (2011).

[37] T. Ding et al., Nonlinear Coherence Effects in TransientAbsorption Ion Spectroscopy with Stochastic ExtremeUltraviolet Free-Electron Laser Pulses, Phys. Rev. Lett. 123, 103001 (2019).

[38] See Supplemental Material at http://link.aps.org/ supplemental/10.1103/PhysRevX.11.031001 for the instrument response function; the production of singly charged cations; the calculation of the cationic absorption spectra, as well as additional such spectra; results from an additional measurement in iodomethane, the extension of the reconstruction method for molecular dynamics to a broader spectral range, which includes Ref. [39].

[39] L. Nahon, P. Morin, and F. Combet-Farnoux, Relaxation of the $n d \rightarrow(n+1) p$ Resonances in Atomic Bromine and Iodine, Phys. Scr. T41, 104 (1992).

[40] R. Moshammer et al., Second-Order Autocorrelation of XUV FEL Pulses via Time Resolved Two-Photon Single Ionization of He, Opt. Express 19, 21698 (2011).

[41] K. Tiedtke, J. Feldhaus, U. Hahn, U. Jastrow, T. Nunez, T. Tschentscher, S. V. Bobashev, A. A. Sorokin, J. B. Hastings, S. Möller, L. Cibik, A. Gottwald, A. Hoehl, U. Kroth, M. Krumrey, H. Schöppe, G. Ulm, and M. Richter, Gas Detectors for X-Ray Lasers, J. Appl. Phys. 103, 094511 (2008).

[42] J. N. Cutler, G. M. Bancroft, D. G. Sutherland, and K. H. Tan, Chemical Dependence of Core-Level Linewidths and Ligand-Field Splittings: High-Resolution Core-Level Photoelectron Spectra of I 4D Levels, Phys. Rev. Lett. 67, 1531 (1991).

[43] J. N. Cutler, G. M. Bancroft, and K. H. Tan, Ligand-Field Splittings and Core-Level Linewidths in I 4D Photoelectron Spectra of Iodine Molecules, J. Chem. Phys. 97, 7932 (1992). 
[44] D. M. Holland, I. Powis, G. Öhrwall, L. Karlsson, and W. von Niessen, A Study of the Photoionisation Dynamics of Chloromethane and Iodomethane, Chem. Phys. 326, 535 (2006).

[45] A. Cartoni, A. R. Casavola, P. Bolognesi, S. Borocci, and L. Avaldi, VUV Photofragmentation of $\mathrm{CH}_{2} \mathrm{I}_{2}$ : The $\left[\mathrm{CH}_{2} \mathrm{I}-\right.$ $\mathrm{I}]^{\bullet+}$ Iso-diiodomethane Intermediate in the I-Loss Channel from $\left[\mathrm{CH}_{2} \mathrm{I}_{2}\right]^{\bullet+}$, J. Phys. Chem. A 119, 3704 (2015).

[46] J. Schirmer, A. B. Trofimov, and G. Stelter, A Non-Dyson Third-Order Approximation Scheme for the Electron Propagator, J. Chem. Phys. 109, 4734 (1998).

[47] D. M. P. Holland, A. W. Potts, L. Karlsson, I. Novak, I. L. Zaytseva, A. B. Trofimov, E. V. Gromov, and J. Schirmer, An Experimental and Theoretical Study of the Valence Shell Photoelectron Spectrum of Bromochlorofluoromethane, J. Phys. B 43, 135101 (2010).

[48] S. Banerjee and A. Y. Sokolov, Third-Order Algebraic Diagrammatic Construction Theory for Electron Attachment and Ionization Energies: Conventional and Green's Function Implementation, J. Chem. Phys. 151, 224112 (2019).

[49] A. Mandal, P. J. Singh, A. Shastri, and B. N. Jagatap, Electronic State Spectroscopy of Diiodomethane $\left(\mathrm{CH}_{2} \mathrm{I}_{2}\right)$ : Experimental and Computational Studies in the

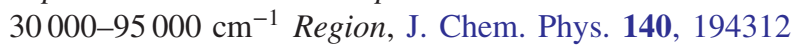
(2014).
[50] Y. Liu, S. L. Horton, J. Yang, J. P. F. Nunes, X. Shen, T. J. A. Wolf, R. Forbes, C. Cheng, B. Moore, M. Centurion, K. Hegazy, R. Li, M.-F. Lin, A. Stolow, P. Hockett, T. Rozgonyi, P. Marquetand, X. Wang, and T. Weinacht, Spectroscopic and Structural Probing of Excited-State Molecular Dynamics with Time-Resolved Photoelectron Spectroscopy and Ultrafast Electron Diffraction, Phys. Rev. X 10, 021016 (2020).

[51] E. Appi et al., A Synchronized VUV Light Source Based on High-Order Harmonic Generation at FLASH, Sci. Rep. 10, 6867 (2020).

[52] A. A. Lutman, R. Coffee, Y. Ding, Z. Huang, J. Krzywinski, T. Maxwell, M. Messerschmidt, and H.-D. Nuhn, Experimental Demonstration of Femtosecond Two-Color X-Ray Free-Electron Lasers, Phys. Rev. Lett. 110, 134801 (2013).

[53] E. Schneidmiller et al., Two-Color Operation of FLASH2 Undulator, in Proceedings of FEL'19, Free Electron Laser Conference No. 39 (JACoW Publishing, Geneva, Switzerland, 2019), pp. 168-171.

[54] K. Tiedtke et al., The Soft X-Ray Free-Electron Laser FLASH at DESY: Beamlines, Diagnostics and End-Stations, New J. Phys. 11, 023029 (2009).

[55] J. Duris et al., Tunable Isolated Attosecond X-Ray Pulses with Gigawatt Peak Power from a Free-Electron Laser, Nat. Photonics 14, 30 (2020). 